Cahiers de philosophie de l'université de

\title{
Martin Heidegger : l'art et la pensée
}

Entretien

\section{Martin Heidegger}

Traducteur : Guillaume Fagniez

\section{OpenEdition}

\section{Journals}

Édition électronique

URL : https://journals.openedition.org/cpuc/287

DOI : $10.4000 /$ cpuc.287

ISSN : 2677-6529

\section{Éditeur}

Presses universitaires de Caen

\section{Édition imprimée}

Date de publication : 1 novembre 2018

Pagination : 11-16

ISBN : 978-2-84133-904-4

ISSN : $1282-6545$

Référence électronique

Martin Heidegger, « Martin Heidegger : l'art et la pensée », Cahiers de philosophie de l'université de Caen [En ligne], 55 | 2018, mis en ligne le 01 novembre 2019, consulté le 31 janvier 2023. URL : http:// journals.openedition.org/cpuc/287 ; DOI : https://doi.org/10.4000/cpuc.287

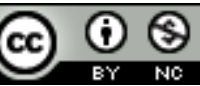

Creative Commons - Attribution - Pas d'Utilisation Commerciale 4.0 International - CC BY-NC 4.0 https://creativecommons.org/licenses/by-nc/4.0/ 


\section{Martin Heidegger : l'art et la pensée ${ }^{1}$}

Heidegger - Nous allons tenter, en partant du point de vue européen qui est le nôtre, de saisir quelques traits essentiels de l'art. La question de savoir si l'art a encore une place quelconque à notre époque est à nos yeux une question d'une extrême urgence. Nous allons commencer le colloque en nous demandant comment ce que nous nommons l'art extrême-oriental se comprend lui-même. De manière tout à fait concrète, nous voulons demander - en supposant admise la diversité du monde extrême-oriental - si l'on peut simplement parler là-bas d'art et d'œuvre d'art au sens où nous l'entendons. Avez-vous au Japon un nom pour l'«art»?

Gundert - On pourrait tout aussi légitimement renverser la question, en demandant si ce que nous appelons art est aux yeux des Asiatiques de l'art, au sens où eux l'entendent. Voilà qui est très souvent contesté au Japon.

Heidegger - Il faudrait, pour apporter une réponse, interroger le concept même de l'art. Nous nous limitons ici, pour notre part, à une réflexion provisoire. Y a-t-il en japonais un mot pour ce que nous appelons art?

Hisamatsu - Il n'est pas difficile de répondre à cette question. L'art au sens moderne (au sens occidental de l'esthétique) existe au Japon depuis environ soixante-dix ans; il procède d'une traduction. Les Japonais ont repris tous les concepts occidentaux en employant pour les restituer d'anciennes racines qui leur sont propres. Pour rendre ces concepts, on a surtout formé des mots composés. Ainsi, Gei signifie originellement l'art entendu comme pouvoir en général, comme habileté. En revanche, le mot composé Gei-jiz rend le concept occidental et esthétique de l'art.

1. Cet entretien du 18 mai 1958 à Fribourg est désormais publié comme $\$ 290$ du tome 16 de l'édition intégrale. Nous remercions Arnulf Heidegger et les éditions Klostermann pour nous avoir donné l'autorisation de publier ces pages. (C) M. Heidegger, «Die Kunst und das Denken. Protokoll eines Colloquiums am 18. Mai 1958», in Gesamtausgabe, Francfortsur-le-Main, V. Klostermann, t. 16, Reden und andere Zeugnisse eines Lebensweges, 200o, p. 552-557. 
Heidegger - Qu'y avait-il auparavant? Est-ce une image qu'on voyait là-bas dans une œuvre d'art? Quelle expérience y faisait-on originellement de l'art, avant de reprendre le concept européen? C'est cela qui doit nous intéresser.

Hisamatsu - Il y a un autre vieux mot pour "art»; un vieux mot japonais, d'un sens plus profond, qui n'a pas subi l'influence du sens européen. C'est Gei-do: la voie de l'art. Do, c'est le Tao chinois, la voie, mais pas seulement au sens d'une méthode; il est profondément et intimement lié à la vie, à notre manière à nous d'être. L'art a ainsi une signification décisive pour la vie elle-même.

Vietta - Cette voie de l'art, le bouddhisme zen y voit-il quelque chose de nécessaire? Et d'abord, l'art a-t-il une nécessité quelconque pour le zen? Pourquoi l'art s'appelle-t-il voie? En quoi le zen a-t-il en général besoin de l'art?

Hisamatsu - Pouvoir, cela a une double signification dans l'art zen : en un premier sens, l'homme est conduit par ce pouvoir de la réalité à l'origine de la réalité; l'art est une voie par laquelle l'homme pénètre jusqu'au cœur de l'origine; en un autre sens, l'art est ce grâce à quoi l'homme, ayant fait irruption dans l'origine, revient vers la réalité. La véritable essence de l'art zen consiste dans ce retour. Ce retour lui-même n'est pas autre chose que l'œuvrer, le se-mettre-en-œuvre de la vérité zen elle-même. L'origine de la réalité dont je parle ici, c'est la véritable vie originelle, le véritable soi, et pour ainsi dire le détachement divin qui délivre de tout lien, le fait d'être affranchi de toute contrainte ayant trait à la forme. Être affranchi de cette manière, nous le nommons également Rien. Ces appellations disent toutes la même chose.

Gundert - Ainsi, il y a dans le zen deux voies: d'abord la voie au sens négatif, sur laquelle la réalité est niée. Obtenir le positif présuppose de passer par ce négatif. Aller à rebours en revenant de ce Rien, faire advenir la part vive: voilà l'essentiel de l'art zen.

Hisamatsu - L'essentiel de l'art zen, ce n'est pas tant d'obtenir l'origine, mais bien plutôt que celle-ci vienne d'elle-même à apparaître. Ce que l'essence du zen recèle de positif consiste en ce jaillissement de l'origine, où c'est l'origine elle-même qui se produit. C'est ainsi qu'œuvre la vérité zen. L'essence du zen, en tant que voie, ne consiste pas dans l'aller, mais bien dans le revenir.

Heidegger - J'aimerais rappeler un entretien que j'ai eu à Vienne avec M. Hisamatsu, qui pourrait nous faire accomplir encore un pas en avant dans la question qui nous occupe ici. L'art européen est caractérisé en son essence par la représentation. Représentation, eidos, rendre visible. 
L'œuvre d'art est une configuration qui met en image, elle rend visible. Dans le monde extrême-oriental en revanche, la représentation est un obstacle, et tout ce qui ressortit à l'image, l'image qui rend visible, a le sens d'un obstacle.

Hisamatsu - Tant que l'homme s'achemine vers l'origine, l'art en tant que représentation de ce qui est porteur d'image est un obstacle. Mais s'il a fait irruption au cœur de l'origine, alors rendre visible (soit le propre même de l'eidétique) ne saurait plus faire obstacle; au contraire, rendre visible, c'est alors faire apparaitre la vérité originelle même.

Heidegger - Ce qui est écrit, ce qui est dessiné n'est pas uniquement un obstacle qui barre le chemin, mais aussi bien ce qui lève l'obstacle, ce grâce à quoi le soi se met en mouvement vers l'origine.

Hisamatsu - Une œuvre d'art zen est belle lorsque le fond primordial y parle de manière vivante. Devient alors possible que ce fond, en s'épanouissant, apparaisse aussi à celui qui regarde.

Heidegger - Dans l'art d'Extrême-Orient, il ne s'agit pas de produire quelque chose d'objectif qui ferait effet sur le spectateur. L'image n'est pas non plus un symbole, elle n'est pas faite pour porter un sens; ce que j'accomplis en peignant, en écrivant, c'est bien plutôt le mouvement d'aller vers le soi.

Hisamatsu - C'est bien cela: l'essence d'une ligne dessinée ne se trouve pas en quelque caractère symbolique, elle consiste au contraire dans le mouvement. Je suis entièrement d'accord avec cette manière de comprendre le mouvement. L'œuvre d'art n'est pas un objet derrière lequel il y aurait une signification ou un sens; elle est bien plutôt immédiate mise en œuvre et mouvement. Seulement, tant qu'il s'agit de parvenir au cœur du mouvement même de l'origine, on n'est plus, ou bien l'on n'est pas encore dans l'origine. En revanche, si l'on est dans l'origine, alors le mouvement se meut de lui-même.

Müller - Pour récapituler, et pour autant que j'aie compris, je dirais volontiers ceci: ce mouvement n'a lieu que si l'on a déjà fait irruption au cœur de l'origine. Le mouvement lui-même n'est pas une propédeutique. L'art authentique ne surgit qu'après avoir atteint l'origine. L'art authentique ne naît pas du mouvement qui nous mène en l'origine, mais bien du mouvement que l'origine accomplit en venant au-devant de nous.

Hisamatsu - En Occident, l'origine est de quelque manière que ce soit un étant, quelque chose qui relève de l'eidétique. Dans le zen, l'origine est le sans-forme, le non-étant. Ce "non» n'est cependant pas une simple négation. Ce Rien est quitte de toutes formes, et c'est pourquoi il peut, au 
titre de ce qui est entièrement affranchi par rapport à la forme, se mouvoir en toute liberté, il peut se mouvoir toujours et partout. C'est en ce libre mouvement que consiste le mouvement à partir duquel l'œuvre d'art est produite.

Heidegger - Ce vide n'est pas le Rien négatif. Si nous entendons le vide comme un concept spatial, alors il nous faut dire que le vide de cet espace est précisément ce qui met en place et aménage un espace, ce qui rassemble et recueille toutes les choses.

Hisamatsu - Je suis entièrement d'accord avec cette idée de mise en place et d'aménagement d'un espace. Cet espacement ne peut être que libre de toute attache. Il faut le soustraire à toute notion d'objectivité ou de validité. Il est le libre œuvrer de la vie qui anime la vérité zen elle-même. La beauté d'une œuvre d'art zen consiste en ce que le sans-forme entre en présence à même ce qui est image, quel qu'il soit. Sans l'entrée en présence du soi libre de forme dans quelque chose qui porte une forme, l'œuvre d'art zen est impossible. Dans le zen, la beauté doit toujours être pensée en relation avec la liberté du soi originel.

Bröse - Ce qui vient d'être évoqué, c'est aussi, pourrait-on dire, ce à quoi aspirent les arts plastiques en Occident. L'œuvre d'art, telle que la vise aujourd'hui l'artiste, n'est pas un symbole, mais le mouvement même qui rend manifeste ce qu'il y a derrière les choses elles-mêmes. L'art emprunte aujourd'hui trois directions: celle du géométrisme, celle de l'absence complète de forme, celle de la position d'un signe. Les arts plastiques modernes veulent mettre de côté toute signification, tout sens. Le but recherché, ce n'est pas quelque chose qui signifie, ni une chose quelconque au sein d'un espace, mais en quelque sorte l'espace lui-même en tant qu'il donne place en rassemblant. En ce sens, Klee est encore un faiseur d'images porteuses de sens. Il y a encore chez lui une instance objective, et non le seul se-mettre-en-œuvre de l'artiste.

Heidegger - Ce que vous dites de Klee est-il vraiment exact? Je ne le crois pas. Mais en admettant qu'il en aille de l'art moderne comme vous avez dit, que reste-t-il une fois le symbolique surmonté? Quelle sorte de monde est-ce là? Il n'est pas permis de passer sous silence cette différence: ce que nous sommes, dans le meilleur des cas, encore en train de chercher ici, au Japon est déjà là, autrement dit les Japonais l'ont déjà.

Hisamatsu - La peinture abstraite: l'essence de son abstraction consiste dans le fait que le peintre se dirige vers l'anéantissement de toute forme. Ce mouvement qui mène au-delà des formes est encore lié à la forme, précisément parce qu'il cherche encore quelque chose par-delà la forme. 
La peinture zen en revanche suit un mouvement exactement inverse. Ce dont il s'agit là, c'est la manière dont le soi libre de forme se montre en venant au-devant de nous.

Müller - Une autre différence à ne pas négliger, c'est précisément que la peinture japonaise peint des choses, tandis que la peinture abstraite n'en peint pas.

Bröse - La peinture non figurative elle-même, qui ne prend son élan que dans la forme et le mouvement, retombe spontanément dans des formes, dans des choses tout à fait similaires aux choses réelles. Elles n'en sont pourtant pas des reproductions. Elles naissent de la tentative d'acquérir certaines dimensions du Rien.

Hisamatsu - Nous autres, Japonais, nous sommes d'emblée en bonne intelligence avec la peinture abstraite.

Alcopley - J'aimerais demander s'il faut tenir une certaine calligraphie du groupe Bokujin pour de l'art zen. Ce groupe est très proche de la peinture abstraite moderne.

Hisamatsu - Je ne souhaite pas m'exprimer sur ce groupe, car je ne veux pas le critiquer ici.

Alcopley - Quels sont les critères qui permettent de juger si une œuvre d'art provient ou non de l'origine?

Hisamatsu - Cela ne peut s'apercevoir qu'à partir de l'origine même.

Bröse - Il y a chez nous différents domaines de l'art, comme la peinture, la musique, etc. Y a-t-il aussi au Japon différents arts? Et qu'en est-il de ces arts qui d'après nos concepts n'en sont pas, par exemple la composition florale, la cérémonie du thé, etc. ? Y a-t-il là aussi des arts à proprement parler?

Hisamatsu - La beauté des œuvres d'art zen, l'essence de cette beauté consiste dans le libre mouvement du soi originel. Si ce mouvement vient à paraître grâce à une forme, il y a bien là une œuvre d'art. Cette venue en présence n'est pas limitée au seul domaine de ce qui est porteur d'une forme au sens artistique du terme. Bien au contraire, la plus haute beauté se trouve là où ne restent plus ni forme ni structure. Dans le zen, l'art n'est donc pas limité à des domaines particuliers. Le mouvement dont nous parlons peut au contraire venir partout en présence.

[Anonyme] - Y a-t-il dans l'art zen une technique, un apprentissage? Cet art s'apprend-il ? Ou un tel art n'existe-t-il qu'à partir de l'expérience zen? Ou encore, est-ce une question de don? 
Hisamatsu - Lorsqu'un artiste est parvenu à l'expérience, il peut aussi trouver le moyen de faire que la vérité vienne à apparaitre. L'apprentissage technique de l'artiste consiste précisément à trouver le moyen approprié. $Y-\mathrm{Y}$ a-t-il un art zen moderne?

Hisamatsu - En de multiples sens. Là où le zen est vivant, l'art est partout.

Heidegger - Il est devenu clair que nous ne pouvons pas, en usant de nos représentations (notamment de notre représentation d'un chemin direct et continu), atteindre le point où les Japonais se trouvent déjà. J'aimerais conclure par un koan, le koan préféré de Maître Hakuin: [levant une main] «Écoute le son du battement d'une seule main!».

Traduction de Guillaume FAGNIEZ 